\title{
Photonic Band Gaps and Unusual Photon Transport in Hyperuniform Disordered Structures Marian Florescu $^{1,}$ Salvatore Torquato ${ }^{2}$ and Paul J. Steinhardt ${ }^{3}$
}

\author{
${ }^{1}$ Advanced Technology Institute and Department of Physics, University of Surrey, Guildford, Surrey GU2 7XH, United Kingdom \\ ${ }^{2}$ Department of Chemistry, Princeton University, Princeton, NJ, USA \\ ${ }^{3}$ Department of Physics, Princeton University, Princeton, NJ, USA \\ m.florescu@surrey.ac.uk
}

Abstract: We demonstrate that hyperuniform disordered structures support electromagnetic states with very different transport properties, ranging from Bloch-like modes to diffusive states with characteristic time scales almost two-orders of magnitude larger.

(C2012 Optical Society of America

OCIS codes: $130.5296,130.7408,160.5293,160.5298$

Recently, a new class of disordered materials with large complete photonic band gaps (PBG), namely, hyperuniform disordered structures [1] has been introduced. Central to this new class of materials is the concept of hyperuniformity, which was first introduced as an order metric for ranking point patterns according to their local density fluctuations [2]. As defined in real space, a point pattern is hyperuniform if the number variance within a spherical sampling window of radius $R$ (in $d$ dimensions), grows more slowly than the window volume for large $R$, i.e., more slowly than $R^{d}$. Hyperuniform structures include not only crystals and quasicrystals, but also constrained disordered structures. The hyperuniform patterns we are considering here are restricted to the subclass of hyperuniform disordered structures. Hyperuniform photonic materials are then constructed by decorating hyperuniform point patterns with dielectric inclusions [1]. Hyperuniform disordered structures are statistically isotropic and possess a constrained randomness such that density fluctuations on large scales behave more like those of ordered solids, like crystals or quasicrystals, than like those of conventional amorphous materials. As a result of this constrained disorder, the materials display an unusual combination of physical characteristics, some associated with random structures, such as statistical isotropy and multiple scattering resulting in localized states, and others associated with periodic structures, such as large and robust PBGs. The PBG formation in disordered photonic materials results from a combination of global hyperuniformity, uniform local topology, and short-range geometric order, and recent experiments performed on microwave hyperuniform structures have demonstrated the existence of PBGs in these materials [3]. But the subtle interplay between constrained large-scale fluctuations and short-range geometric order not only gives rise to large and robust PBG, but also strongly modifies the properties of the photon transport.

In this study we analyze the properties of 2D hyperuniform disordered photonic materials for TM polarized radiation. Employing finite-difference-time domain calculations we characterize the isotropy of the PBG and the novel features in the photon transport through these structures. While the present study deals only with TM polarized radiation, qualitatively similar results can be obtained for TE polarized radiation for structures designed using the constrained optimization protocol developed in Ref. [1]. The structures analyzed here are generated by decorating hyperuniform point patterns (typical sample contains $N=500$ points) with dielectric cylindrical scattering centers with a dielectric constant of $\varepsilon=11.56$ and radius $r / a=0.189$ chosen to maximize the size of the photonic band gap. Here, we have introduced a length scale $a=L / N^{1 / 2}$, such that the hyperuniform patterns have point density $1 / a^{2}$. All the hyperuniform point patterns used in this study are obtained using the previously developed generalized coordinate method and are characterized by an hyperuniformity order parameter $\chi=0.5$. It is essential to note that PBGs for these disordered structures are equivalent to the fundamental band gap in periodic systems, i.e., the spectral location of the gap is determined by the resonant frequencies of the scattering centers and always occurs between band $N$ and $N+1$, with $N$ precisely the number of points per unit cell. Typical PBG size is $\Delta \omega / \omega_{C}=37 \%$, where $\omega_{C}$ is the central frequency of the gap, and varies only by few percent among different configuration with the same value of the hyperuniformity order parameter.

In general, the propagation of the radiation in a dielectric material is strongly dependent on the refraction index contrast the shape of the scatters and the presence or absence of structural order. The 
periodic order present in photonic crystals give rise unique effects, such as the complete absence of photonic transport in the photonic band gap spectral region and the propagation without losses and anomalous dispersion for propagation in the allowed regions. On the other hand, conventional disordered materials are usually white and opaque and the radiation propagates diffusively through the structure. In hyperuniform dielectric structures, the presence of local geometrical order and the reduced large-scale spatial fluctuations enable well defined localized resonances, which strongly affects the radiative properties of the structure. It has been demonstrated that analogous to the case of photonic crystals, for both quasiperiodic [4] and hyperuniform disordered [1] PBG materials, the electric field for the states below the photonic band gap is well localized in the cylinders (the high dielectric component), while the electric field for states above the band gap is localized in the air fraction. To elucidate the influence of localized resonance and their localization properties on the photon transport, we analyze the electric field distribution for two propagating modes in the hyperuniform structure. Plane-wave like narrow pulses are sent at normal incidence and the field is recorded at different time intervals. We focus for two propagating modes with frequencies below $\left(\omega_{1} a / 2 \pi c=0.24\right)$ and above $\left(\omega_{2} a / 2 \pi c=0.48\right)$ of the photonic band gap. Both modes are characterized by sizable transmission, $T_{1}=0.8$ and $T_{2}=0.35$, respectively. As shown in Fig. 1, for the mode below the band gap the photon transport proceeds in Bloch-like fashion by scattering on effective planes separated by a well-defined average distance. In contrast, the photon transport for states above the PBG proceeds in a diffusive manner. The insets in Fig. 1 show the electric field distribution at two arbitrary positions in the sample demonstrate that the mode below the PBG excites resonances similar to the first Mie resonance of the isolated cylinders (with the field localized mostly inside the dielectric material), whereas the mode above the PBG excites resonances similar to the second Mie resonance with a nodal plane passing through the dielectric cylinders.

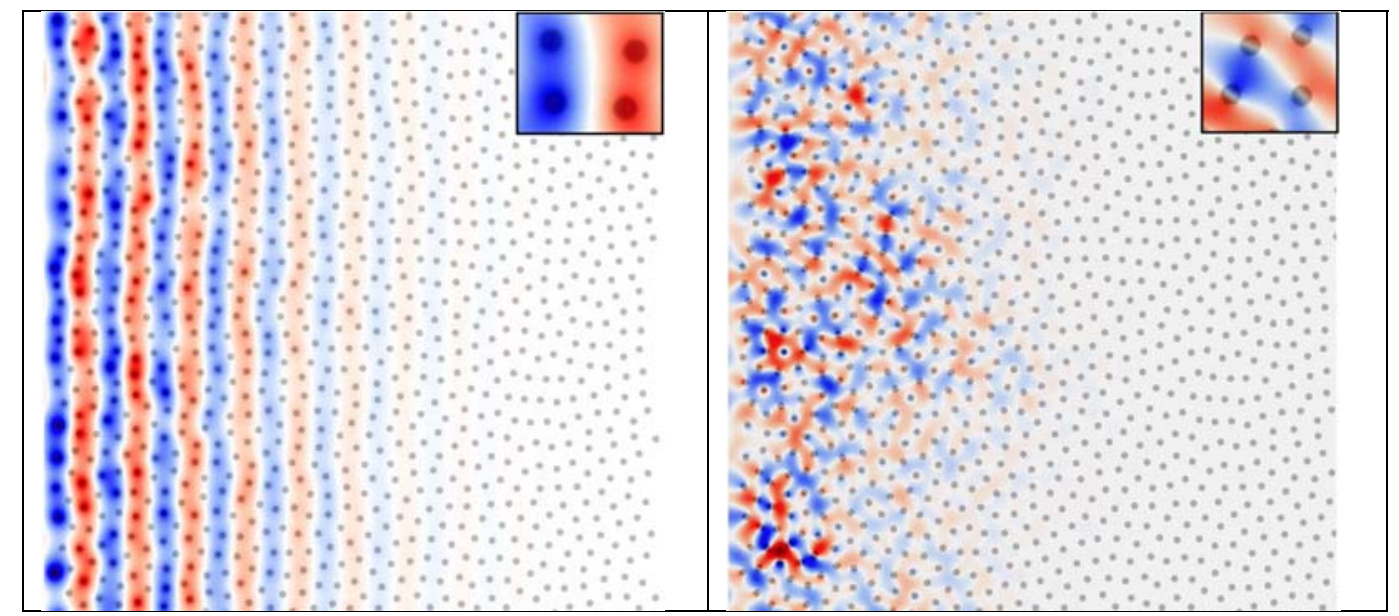

Figure 1. Field distribution for two modes propagating modes with frequencies left) below $\left(\omega_{1} a / 2 \pi c=0.24\right)$ and right) above $\left(\omega_{2} a / 2 \pi c=0.48\right)$ of the PBG. Also pictured are insets at two arbitrary positions in the sample.

In summary, we analyzed the transport of the electromagnetic radiation in hyperuniform disordered structures and shown that the hyperuniformity-mediated strong scattering not only redistributes the photonic states and gives rise to large and robust photonic band gaps, but also strongly affects the photon transport in these materials. We have demonstrated that these materials support electromagnetic states with very different transport properties, ranging from modes in which the photon transport proceeds in Bloch-like fashion by scattering on effective planes separated by a well-defined average distance, to states where the photon transport proceeds in a diffusive manner with characteristic time scale almost two-orders of magnitude larger.

[1] M. Florescu, S. Torquato, and P. Steinhardt, PNAS 106, 20658 (2009)

[2] S. Torquato and F. H. Stillinger, Phys. Rev. E 68, 041113 (2003).

[3] W. Man, M. Florescu, K. Matsuyama, P. Yadak, S. Torquato, and P. J. Steinhardt, Conference on Lasers and Electro-Optics (Optical Society of America, Washington, DC, 2010), CThS2.

[4] M. Florescu, S. Torquato, and P. J. Steinhardt, Phys. Rev. B 80, 155112 (2009). 